\title{
Business Process Design from Virtual Organization Intentional Models
}

\author{
Luz María Priego-Roche ${ }^{1}$, Lucinéia Heloisa Thom ${ }^{1,2, \star}$, Agnès Front ${ }^{1}$, \\ Dominique Rieu ${ }^{1}$, and Jan Mendling ${ }^{3}$ \\ 1 Laboratoire LIG, Equipe SIGMA, BP 72, 38402 Saint Martin d'Hères, France \\ \{Luz-Maria.Priego-Roche, Dominique. Rieu, Agnes. Front\}@imag. fr \\ 2 Universidade Federal do Rio Grande do Sul, Instituto de Informática, \\ Av. Bento Gonçalves Porto Alegre, RS, Brasil - CEP 91501-970 \\ lucineia@inf.ufrgs.br \\ 3 Wirtschaftsuniversität Wien, Augasse 2-6, 1090 Vienna, Austria \\ jan.mendling@wu.ac.at
}

\begin{abstract}
Virtual Organizations (VO) have emerged as a new type of interorganizational relationship for dealing with emerging challenges. The information system support for a VO poses new challenges to system design. Recent works define three levels of abstraction, namely an intentional level, an organizational level, and an operational level. For such a staged design, it is fundamental that artifacts defined on the different layers are consistent with each other. We address this problem based on a transformation approach. We illustrate the transformation from the intentional level towards the organizational level based on the $360^{\circ}$ VisiOn and the BPMN process modeling language. The approach has been implemented in a prototype and validated using a case study from a regional stockbreeder union in Mexico.
\end{abstract}

Keywords: Virtual organizations, intentional modeling, organizational modeling, workflow activity patterns.

\section{Introduction}

Virtual Organizations (VOs) have emerged as a new type of inter-organizational relationship to deal with emerging challenges such as new competitors, new markets, or new customer needs. A VO is an alliance for integrating competencies and resources from independent companies, potentially geographically dispersed [13]. This integration has to be facilitated by appropriate information system support. A new challenge in this context is the need for methods for streamlining discussions among participant organizations, examining stakeholders requirements and analyzing the environment [2].

Different approaches of requirement elicitation can be used for modeling traditional organizations including $i^{*}[23]$, KAOS [20], Maps [15], and scenarios [9]. The $\mathrm{e}^{3}$ value approach [5] has been designed for service-oriented collaboration. In our work, the $360^{\circ}$ VisiOn approach has been addressed to VOs . This approach defines three levels

\footnotetext{
* Special thanks to the Coornedçāo de Aperfeiçoamento de Pessoal de Nível Superior from the Brazilian government
} 
of abstraction for building the information system of a VO. First, the intentional level describes the "problem space" by identifying the objectives and collaboration. Second, the organizational level specifies the "solution space" by establishing the business process. Third, the operational level defines the "implementation space" by developing the information system. A central challenge is to establish a comprehensive specification which is consistent between the intra-organizational, the inter-organizational, and the extra-organizational perspective.

In this paper, we address the problem of defining business processes on the organizational level which are consistent with models on the intentional level. Our approach builds on a pattern-based mapping between the constructs of the first and the second level. The transformation requirements are aligned with the concept of workflow activity patterns (WAPs) in order to achieve semantically rich process models. The approach is based on a $360^{\circ}$ VisiOn modeling tool as a transformation to BPMN. We use the case of a regional stockbreeder union in Mexico to demonstrate the applicability of our approach.

The paper is structured accordingly. Section 2 gives an introduction to the $360^{\circ} \mathrm{Vi}$ siOn framework, Section 3 describes the intentional level of the framework and its underlying metamodel. Section 4 gives an overview of BPMN and defines the transformation rules from $360^{\circ}$ VisiOn to BPMN. Section 5 introduces the WAPs for the enrichment process at the organization level. Section 6 shows the application of the approach for the case of the stockbreeder union in Mexico. Section[7 discusses the results in the light of related work. And finally, Section 8 summarizes our proposals and prospects for future study.

\section{Overview of the $360^{\circ}$ VisiOn}

The $360^{\circ}$ VisiOn proposes a framework for eliciting VO's requirements from a vertical and a horizontal approach [13]. The levels of analysis are (see Figure 1]a):

- Vertical: Intentional (emphasizing the alliance, collaboration and common objectives), Organizational (formalizing the business processes) and Operational (executing the BP).
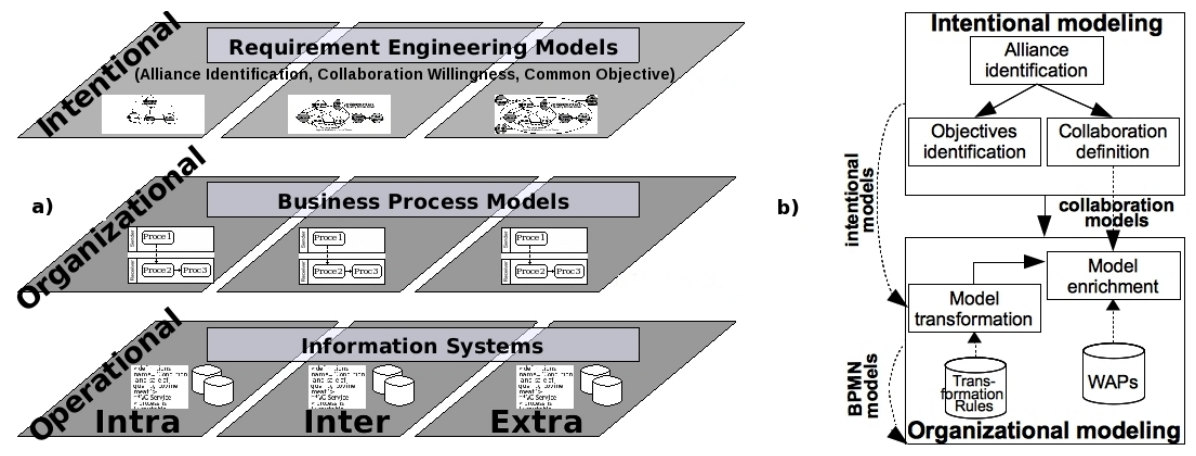

Fig. 1. The $360^{\circ}$ VisiOn and building the VO's business processes 
- Horizontal: Intra-organizational (focusing on the relationships inside each organization forming the VO), Inter-organizational (focusing on the relationships among the organizations forming the VO) and Extra-organizational (focusing on the organizations of the external environment).

Figure 1 $\mathrm{b}$ shows a simplified view of the intentional modeling based on the $360^{\circ}$ VisiOn approach. These intentional models are the input for building the BPMN diagrams. We use the latter as a target model notation at the organizational level, since it is a stancedard notation being adopted by the business community as well as by many BPM tools. We carry out a transformation process based on transformation rules. As seen in Figure1 $\mathrm{b}$, in this paper the collaboration diagrams are improved with an enrichment process based on WAPs.

\section{The Intentional Level}

The characterization of the $360^{\circ}$ VisiOn is composed of the following aspects that guide the intentional models development and are shown in Figure 1

Alliance Identification of the agreement, actors and services offered. The alliance establishes the relationship preserving organization's independence for continuing their own projects. Actors (e.g. stakeholders, users, organizations) identification is a recommended way for starting eliciting IS requirements [6]. Services identification defines the general output expected from these actors and their role in the service generation.

Collaboration Willingness characterizes the compromises each Member Organization plans to give to the $\mathrm{VO}$ in terms of its availability to the new relationship, the investments willing to make, the elements to be coordinated and the regulation of the expected behavior that would assure VO members good performance.

Common Objective characterizes the shared goal and the directions to be followed for achieving it. The latter could answer customer's needs (integral services), satisfy companies' objectives (to share costs, to create more effective processes), make new business (markets, products or services) or confront difficulties (absence of knowledge).

The simplified UML 11 meta-model of the three aspects composing the $360^{\circ}$ VisiOn is shown in Figure2. To illustrate them, two aspects are instantiated with graphical and textual models, the implementation was done with a software tool prototype [13] shown in Figures 3 and 4 ,

The Alliance Identification states a set of organizations forming a VO by subscribing an alliance to offer a service. To explore this aspect, different driving forces are considered: at the Inter level, the end consumer as the reason of the organization network existence, at the Intra level, each organization's functional actor that takes part of the service delivery and at the Extra level, the environmental organizations that influence the service.

Inspired by [1], Figure 3 presents the Inter and Extra views. Internal Organizations participate in the service as Member Organization that agrees to join the alliance and accordingly to acquire compromises and rewards beyond the service offered (e.g. Stockbreeders, Stockbreeders Associations, Freight \& Trucking, Slaughter House and Meat

${ }^{1}$ Unified Modeling Language. 


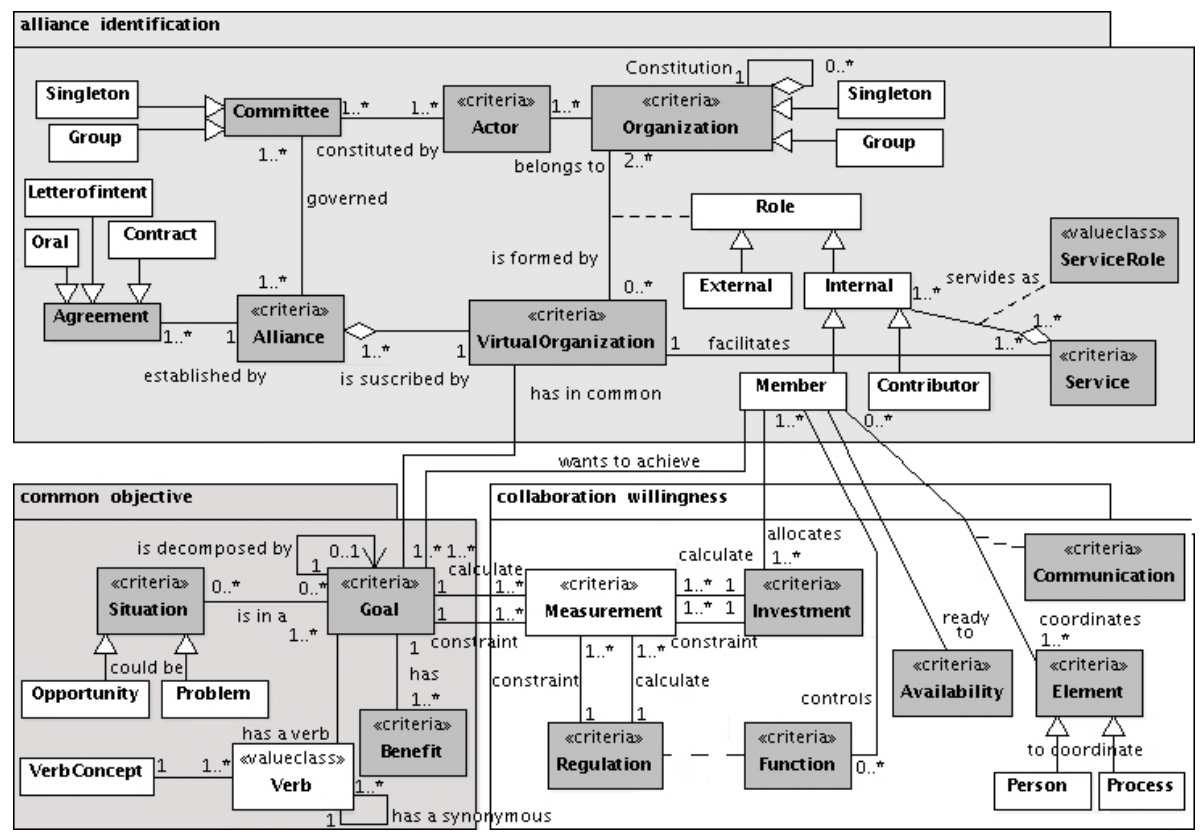

Fig. 2. The $360^{\circ}$ VisiOn meta-model

Marketing), or as Contributor Organization that does not agree to join the alliance, however interacts directly with the VO either soliciting or rendering services. A Contributor Organization may become a Member Organization that may extend the VO boundaries (e. g. Supermarket Chains and Meat Consumers). The Member Organizations form the VO Regional Stockbreeders Union of Tabasco (UGRT) are delimited by a dotted oval. External Organizations influence the service like Government Regulators, Meat Importers and Leather Article Producers which have a respective Regulatory, Concurrently, and Complementary role in the Service. Members and Contributors provide one or more services, e.g. Conditioning and Sale of Quality Bovine Meat, detailed in the text model of the VO. These organizations play a service role (as service, direct, indirect, and auxiliary provider, or service consumer), the Service text model shows the Stockbreeder as an Indirect Provider that Provides grass fed cattle livestock.

A VO is subscribed by an alliance based on an agreement which is established orally or written with a letter of intent or a contract. This alliance is governed by a committee which is constituted by actors that belong to an organization. Either organizations and committees are formed by only one (singleton) or more than one (group) of them. Among the information agreed in the alliance is the facade to be expose between the VO and its clients or users: screen (only the VO is viewed), semi-screen (the VO and all or some organizations are viewed) and mirror (only the organizations are clearly viewed). In the Alliance Identification text model, a semi-screen facade was agreed allowing clients to view the UGRT and some of the member organizations.

The Collaboration Willingness arises Member Organizations responsibilities and advantages to the VO. The alliance formed among the Member Organizations allows to 


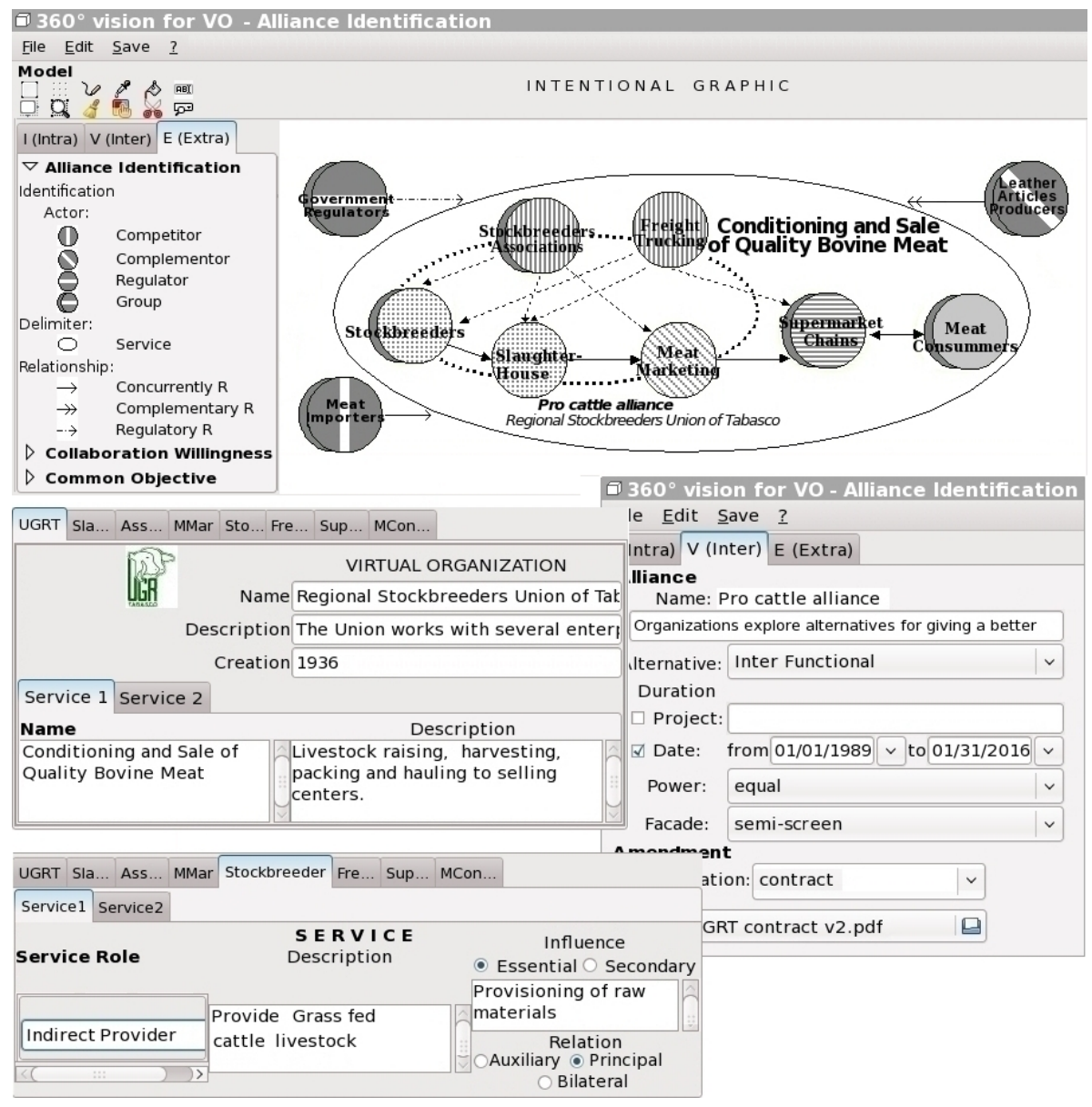

Fig. 3. The Alliance Identification models for the UGRT

explore collaboration not only for service provisioning but to find other means that benefit Member Organizations (e.g. better logistic coordination, resources optimization).

Firstly, from the meta-model each Member Organization is ready to give an availability to the VO in terms of time, priority to the relationship and adaptability to changes. It also allocates one or more investments to the VO. These investments can have one or many measurement to be calculated or to constraint them. A partial view of the investments graphic representation is given in Figure 4 showing a chart where the arcs bind the Stockbreeder Member Organization with the VO. The three investments are represented with dotted squares. The icon inside the square illustrates its type (Assets: financial or material, or Capital: human, relational or organizational[12]). The text model shows a financial asset investment ("Contribution") described as "Give a contribution for each slaughtered cattle" which is fixed by an "Average Cost". This contribution is event-triggered (when the cattle is slaughtered) and has a direct impact on the VO. 


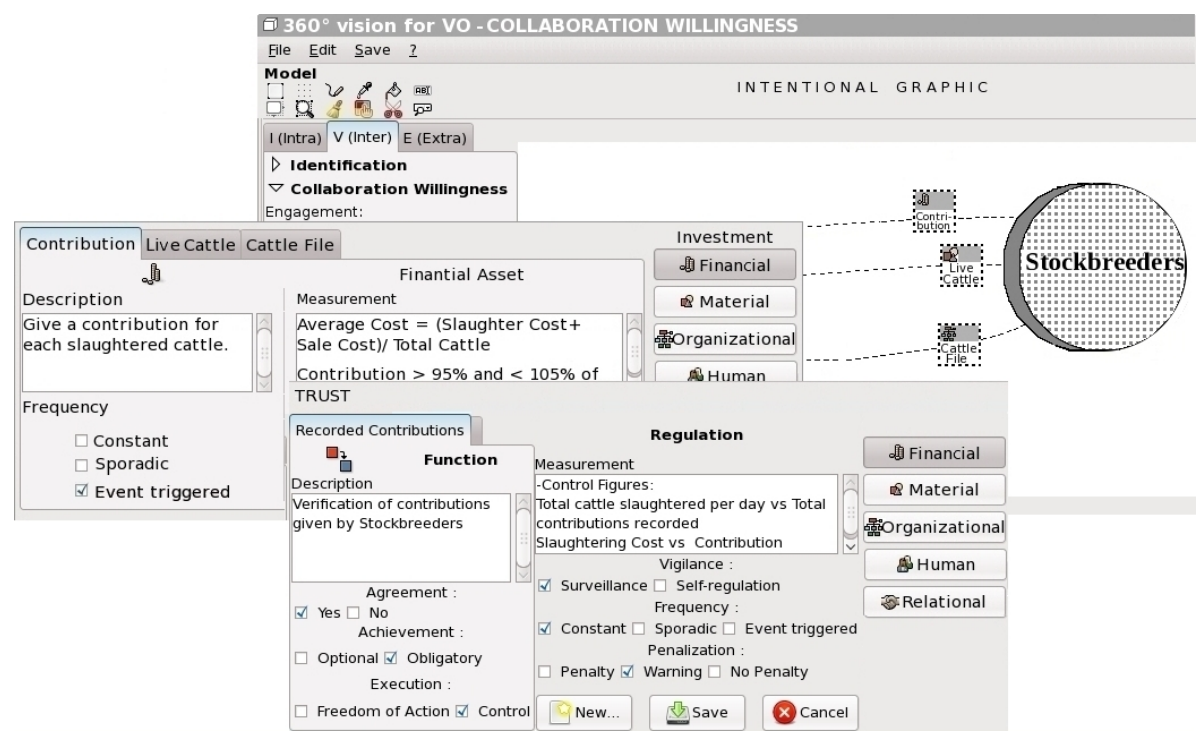

Fig. 4. The Investment models for the UGRT

Secondly, each Member Organization has one or more elements to coordinate. An element can be a person or a process. The elements' communication is described in terms of space (where), time (when) and movement (how) [17].

Finally, Trust fosters an effective collaboration. Each Member Organization may or may not have a function to establish alertness for assuring the compromises well execution. The regulation of these functions can be controlled or not by other Member Organization in a constant, sporadic or event-triggered frequency and in case of violation to these controls a penalization (penalty, no penalty, or warning) can be established. These regulations are quantified through measurement, a set of calculations and constraints. The text model linked to the "Contribution" Investment named "Regulation", consist of a "Verification of contributions given by Stockbreeders". Contributions are constantly surveyed by other organizations and there is a "Warning" in case of miss achievement.

Finally, the Common Objective characterizes the goals all Member Organizations have in common to form a VO (Inter level) and those each Member Organization wants to achieve to justify the alliance (Intra level). A goal is described by a verb, a targeted object and a complement. Goals have one or more expected benefits representing the foreseen goal yield and can be measured by constraints or calculations. Usually, objectives can emerge from a situation that could be classified in opportunity (circumstances favoring the alliance) or problem (difficulties that justify exploring the alliance).

\section{Obtaining Basic Business Processes Models}

The $360^{\circ}$ VisiOn models provide a clearer understanding of a VO from an intentional perspective. Business process modeling allows organizations to understand and 
communicate their internal business processes in a formal or semi-formal graphical notation from an organizational perspective. It also allows organizations to analyze, model, execute, and monitor their business processes. UML, EPC (Event Process Change), and BPMN are alternatives for representing business processes. The transformation from the intentional to the organizational models is a complex task that involves establishing a formal model to review and verify the transformation and selecting the correct equivalent representation between the two different models in order to assure accurate and coherent BPM.

\subsection{Transformation Process: From Intentional to Organizational Models}

Once the alliance is identified (Figure 3), each Member Organization determines the resources willing to give and expecting to receive (Figure 4) as well as the common objective that unifies them for working together. When the intentional level modeling is considered complete, it is required to define the Member Organizations business processes needed by the VO for satisfying the service and contributing to the consented objectives. As stated before, one user defines the intentional level, then $\mathrm{s} / \mathrm{he}$ gives it on to another user to complete the models at the organizational level.

A VO can facilitate many services, where each service is represented with one BPMN diagram. Based on [22] we state a high level business process as the description of a set of one or more linked procedures representing the know how of each organization which collectively satisfy the defined service. The complete BPMN notation from [11] is the basis of the translation from the $360^{\circ}$ VisiOn to BPMN. The translation process can be seen as a partial function $f: \mathbb{C} \longrightarrow \mathcal{P}(B)$ mapping from $360^{\circ}$ VisiOn Criteria domain $\mathbb{C}$ to the BPMN Construct $\mathbb{B}$ domain. The first step of the strategy followed consists in analyzing each criteria of the intentional aspects and mapping it to the more suitable BPMN constructs.

The first step is based on the alliance identification intentional models. Below, we define the rules for assigning these Criteria of the $360^{\circ}$ VisiOn from the simplified UML meta-model given in Figure2 to the BPMN constructors.

\subsection{From the Alliance Identification to BPMN Collaboration Diagram}

The virtual organization is denoted by $V O$ and it facilitates one or more services. Member organizations, contributor organizations and external organizations are denoted by the sets $O_{m}, O_{c}$ and $O_{e}$ respectively. A virtual organization service $V O S$ will be denoted as a tuple $\left(M O_{s}, C O_{s}, E O_{s}, S R_{s}\right)$ where

- $M O_{s}$ is the part of the virtual organization related with members that facilitate the service,

- $C O_{s}$ is the part of the virtual organization related with contributors that facilitate the service and

- $E O_{s}$ is the part of the virtual organization related with externals that influence the service.

- $S R_{S}$ is the collection of Service Roles for the service. 
$M O_{s}, C O_{s}, E O_{s}$ and $S R_{s}$ do not have direct representations in the UML meta-models of Figure 2, nevertheless for formalization purposes, they will be considered as $\mathbb{C}$ constructs that could be built from the UML meta-models. $M O_{s}$ is represented by a tuple $\left(O_{m s}, S R_{m s}\right)$ where

- $O_{m s}=\left\{o_{m s}, \ldots, o_{m s i}\right\} \subseteq O_{m}$, is the set of member organizations that participate in the service, and

- $S R_{m s}=\left\{s r_{m s}, \ldots, s r_{m s i}\right\} \subseteq S R_{s}$ is the set of roles of member organizations in the service and $s r_{m s a}$ corresponds to the service role served by $o_{m s a}$.

$C O_{s}$ is represented by a tuple $\left(O_{c s}, S R_{c S}\right)$ where

- $O_{c s}=\left\{o_{c s 1}, \ldots, o_{c s j}\right\} \subseteq O_{c}$ is the set of contributor organizations that participate in the service, and

- $S R_{c s}=\left\{s r_{c s 1}, \ldots, s r_{c s j}\right\} \subseteq S R_{s}$ is the set of roles of the contributor organizations in the service and $s r_{c s a}$ corresponds to the service role served by $o_{c s a}$.

$E O_{s}$ is a set of external organizations $\left\{o_{e s}, \ldots, o_{e s k}\right\}$.

$S R_{s}$ is a set of service roles $\left\{s r_{1}, \ldots, s r_{n}\right\}$.

Therefore, the rules for assigning the Criteria of the Alliance Identification are:

Rule 1: Each Service facilitated by the VOS is represented by one BPMN Collaboration Diagram which contents are determined by the application of all the following rules, from Rule 2 to Rule 11.

Rule 2: Each $M O_{s}$ is represented by one BPMN Pool contained in a BPMN Process.

Rule 3: Each $C O_{s}$ is represented by one BPMN Pool contained in a BPMN Process.

Rule 4: Each $E O_{s}$ is represented by one BPMN Pool contained in a BPMN Process.

Rule 5: The BPMN Pools obtained by the application of Rules 2 to 4 are included in the representation of the BPMN Collaboration construct.

Rule 6: Each Member Organization is represented by one BPMN Lane which includes a reference to its service role description.

Rule 7: The VO facade is represented by one BPMN Lane inside the $M O_{s}$ BPMN Pool if and only if facade is screen or semi-screen.

Rule 8: Each Contributor Organization is represented by one BPMN Lane which includes a reference to its service role description.

Rule 9: Each External Organization is represented by one Lane which includes a reference to its service role description.

Rule 10: For each ServiceRole there is one Activity Object stereotyped with its RoleName. This Activity Object contains three BPMN constructors: one sub-Process, several sequenceFlows (between organizations within the same pool) and messageFlows (between organizations in different pools).

Rule 11: For each ServiceRole there is a collection of messageFlows, one for each collaboration between service roles associated to organizations in other pools. 


\section{Organizational Level Enrichment}

Based on transformation rules we have obtained the basic BPMN diagram that includes pool, lines, sub-processes and flows constructs needed to represent the service from the Alliance Identification models. To complement the business process development we use recurrent business functions called WAPs and illustrate the enrichment process using one of the collaboration willingness models. We extract the sequence of BPMN Construct from the $\mathbb{B}$ domain that match with the WAPs domain $\mathbb{W}$, where $\mathbb{W} \subseteq \mathbb{B}$.

\subsection{An Overview on Workflow Activity Patterns}

A WAP refers to the description of a recurrent business function (e.g. task execution request, approval) as it can be frequently found in business processes. In this paper we use a subset of the seven activity patterns defined in [19] to enrich the transformations from the intentional to the organizational level of a VO and obtain process models fragments executed by the organizations. The activity patterns comprise the following behavior:

Approval (WAP1): An object (e.g. a document) has to be approved by one or more roles. The evaluation can be executed only once (single approval) or multiple times. In the latter, it can be done in sequence (iterative approval) or in parallel (concurrent approval).

Question-Answer (WAP2): An actor might have a question before or during an activity in the process. The pattern formulates such question, identifies a role who is able to answer it, sends the question, and waits for response (single question-answer). The question can be sent to multiple roles or actors resulting in multiple answers (multiquestion-answer).

Unidirectional Performative (WAP3): A sender requests the execution of an activity from a receiver (e.g. a human) involved in the process. The sender continues execution of his part of the process immediately after having sent the request. A requestor sends an activity execution request to one receiver (Single-Request) or to many (Multi-Request).

Bi-directional Performative (WAP4): A sender requests the execution of a particular activity from another role involved in the process. The sender waits until the receiver notifies him that the requested activity has been performed.

Notification (WAP5): The status or result of an activity execution is communicated to one or more process participants.

Information Request (WAP6): An actor requests certain information from a process participant. S/he continues process execution after having received the desired information.

Decision (WAP7): During process enactment, the execution of one or multiple activities is requested. Depending on the executions results the process continues through one or several branches. The pattern allows to include a decision activity with connectors to different subsequent execution branches (each of them is associated with a specific transition condition if evaluated to true, its branches are selected). 
Figure 5 provides a summary of the WAPs in the BPMN notation. The Approval Pattern (WAP1), for example, starts with a send activity indicating an approval request. A receiver performs the approval and the result of the approval is then sent to the requester. Generally, multiple activity patterns can be composed in a process model using workflow patterns like Sequence, AND-Split, AND-Join or XOR-Split. An empirical study, in which more than 200 real-world process models were analyzed, confirmed the existence of the seven activity patterns [18]19]. The study showed that the analyzed process models can be designed based on the investigated WAPs; i.e., the set of identified activity patterns is necessary as well as sufficient to design the 200 process models, at least at a certain level of granularity.

In [18] the frequency of co-occurring activity patterns in real world process models was also analyzed. In addition a process model mining tool was developed to be used for identifying the activity patterns co-occurrences. The miner allows analyzing process models instead of event logs as proposed in literature. This can be considered as a very important functionality to automatically identify activity patterns co-occurrences (e.g., the pattern pair APPROVAL $\longrightarrow$ NOTIFICATION) in real-world process models. To achieve a precise semantics the patterns had been formalized using Pi-calculus [19]. A process model specified in Pi-calculus can express the dynamic behavior of the process, thus making it possible to verify formal properties of the model like soundness (e.g., absence of deadlocks and livelocks) and model equivalence [8].

\subsection{From the Collaboration Willingness to the BPMN Diagram}

Complementing the BPMN diagram with the Collaboration Willingness models.

Based on the Collaboration Willingness, we illustrate part of the Engagement subaspec that refines the $M O$ 's sub-processes, to do so, we use transformation rules and the WAPs. The Investments each Member Organization makes to the VO is denoted as follow:

- $I_{s}$ is the collection of investments made to the service.

- $I_{m s}=\left\{i_{m s 1}, \ldots, i_{m s p}\right\} \subseteq I_{s}$ is the set of investments of the member organizations in the service and $i_{m s a}$ correspond to the investment made by $o_{m s a}$.

Rule 12: One Investment given by a $M O$ is represented by a BPD Collaboration construct which content is determined by the application of all the following rules, from Rule 13 to Rule 20.

Rule 13: The Frequency is represented by the BPMN Start Event to indicate the investment process start. If the Constant or Sporadic option is selected, a Timer Start Event is chosen to indicate the specific time-date or cycle. If the Event triggered option is selected, the BPMN Conditional Start Event is chosen to indicate that a condition is true.

Rule 14: The Vigilance from the Regulation sub-aspect expresses if the tasks performed by an organization have to be notified to other organizations. Vigilance is represented by a Bi-Directional Performative (WAP4) if the Surveillance option is selected with the variants: requested by one (Single-Request) or many (Multi-Request) organizations. If the Self-Regulation option is selected, the WAP3 Unidirectional Performative is used. 

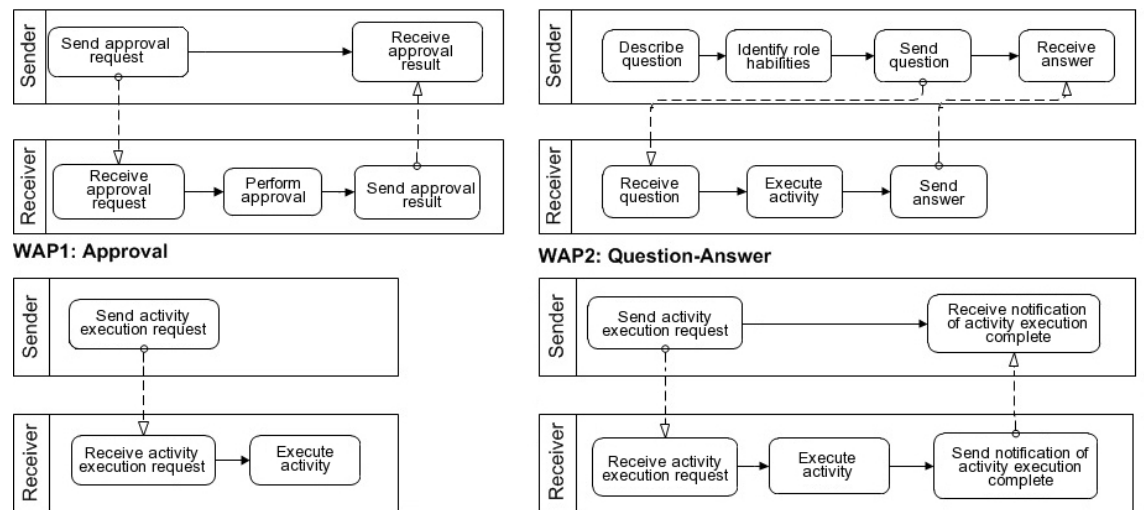

WAP2: Question-Answer

WAP3: Unidirectional Performative
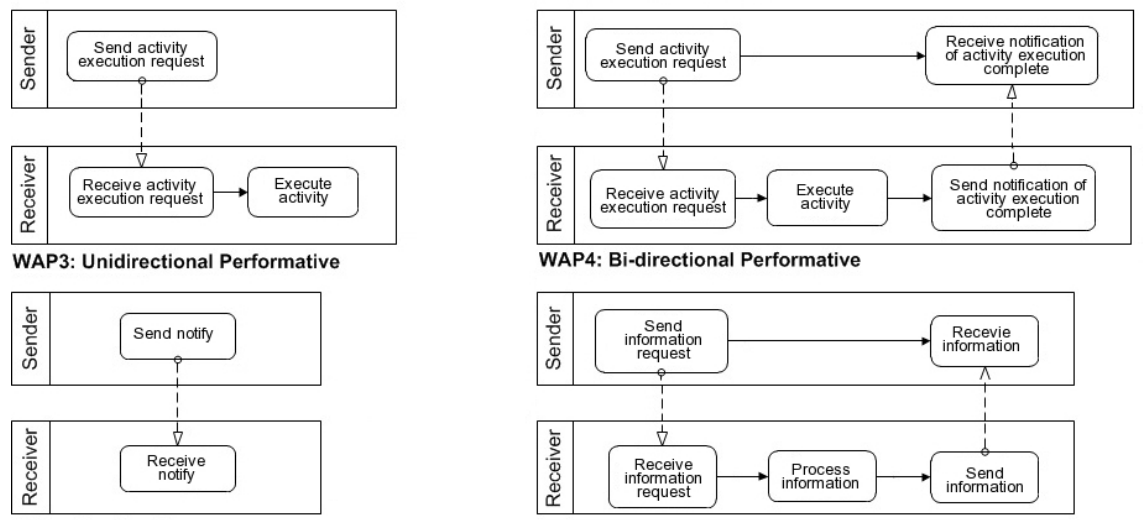

WAP4: Bi-directional Performative

WAP5: Notification
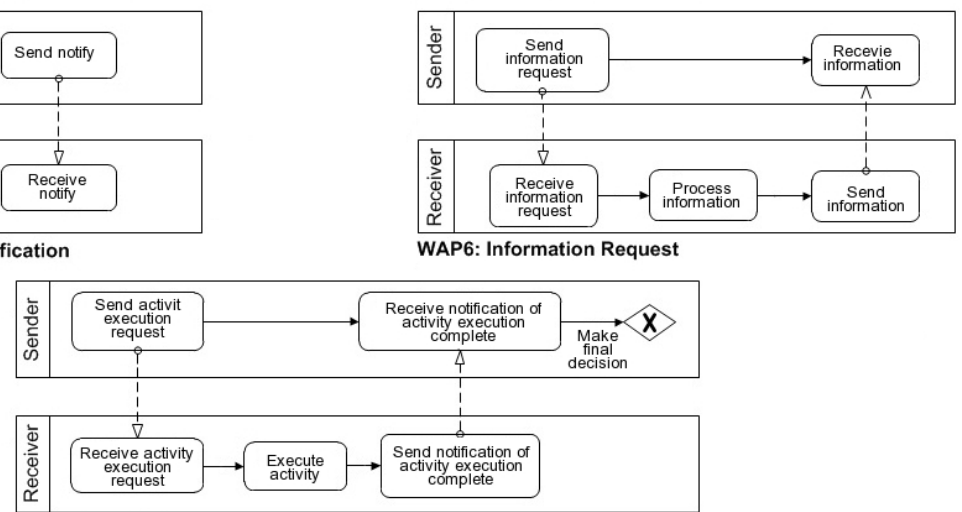

WAP7: Decision

Fig. 5. Summary of Workflow Activity Patterns

Rule 15: The Investment given by the Member Organization is represented with the Bi-Directional Performative (WAP4) if the Surveillance option is selected, with either two variants: requested by one (Single-Request) or many (Multi-Request) organizations. The Unidirectional Performative (WAP3) is represented if the Self-Regulation option is selected.

Rule 16: The Frequency from the Regulation sub-aspect specifies the Vigilance for the BPD Collaboration when the Surveillance option is selected. Constant meaning that Surveillance (notification) is needed in all steps of the process, Sporadic when needed in some steps and Event triggered only when a condition is true.

Rule 17: The Measurements defined in the Investment are used to describe the Business Rules of the BPMN Business Rule Task in the WAP of Rule 14.

Rule 18: The Measurements defined in the Regulation is complemented with the BPMN Business Rule Task in the assigned WAP of Rule 17.

Rule 19: The Data Object of Rule 15 represents the Investment (Assets or Capital) to be given from one organization to another including a descripton reference. 
Rule 20: The Penalization from the Regulation sub-aspect is represented by one BPMN Exclusive Gateway to fork to the Activity object stereotyped with the Penalty or Warning option selected.

Having the BPMN diagram, more semantic can be given to them by:

- Specifying the information of the BPMN Conditional Start Event or the Timer Start Event defined in Rule 13: when cattle slaughtered for the former; or every two weeks, every month, at the end of the year for the latter.

- Defining the Single or Multi Request variants of Rule 14 and 15 (e.g. to which organizations the execution notification has to be sent).

- Establishing the BPMN constructs for controlling the Sporadic or Event triggered Regulation. This is not explicit in Rules 14 and 15 since the WAP4 is used in the collaboration process to represent the "Constant Surveillance".

- Representing Investments with Data Objects, to exchange elements like money, materials which are not available in BPMN.

- Having a multi instance participant for the lane construct (for representing the group of Stockbreeders for example which is not available in BPMN).

\section{Prototypical Implementation for the Stockbreeder Union}

Figure 6 partially shows the alliance identification mapping between the $360^{\circ}$ VisiOn and the BPMN diagrams. Based on the models of Figure 3 it represents the service "Conditioning and Sale of Quality Bovine Meat" of the UGRT case study. On the left hand, the corresponding $f$ definition that creates the collaboration diagram representing the service facilitated by the VO is shown, as well as other constructs. We use the following operators to write these functions: if $<$ condition $>$ then $<$ value $>$ for expressing conditionals, and + for string concatenation. Different font styles are used to distinguish elements in BPMN and XML constructs: bold for operators, typewriter for variables, italic for functions, and ** FOR comments to clarify the construct (for more detail see [14]).

The BPMN diagram corresponding to the mapping of the UGRT service is generated by function (a). Member Organizations (Stockbreeders Associations, Stockbreeders, Slaughterhouse, Freight Trucking and Meat Marketing, Rule 6) and the "semi-screen Facade" Rule 7 are lanes in the pool "UGRT Members" (Rule 2). They are generated by functions (b) and (c). Each Member has a service offered and represented as a sub-Process inter-connected with sequenceFlows (Rules 10 and 11). These sub-Processes are named as the service role, e.g. "Provide grass fed Cattle Livestock" for the Stockbreeder and generated by functions (d) and (e).

Figure 7 shows the BPD diagram corresponding to the mapping of the Stockbreeder Investments shown in Figure 4 according to rules 12 to 20. The Member Organizations involved in this process are the Slaughterhouse, the Stockbreeders, and Meat Marketing. The "Contribution" investment is represented by this collaboration diagram (Rule 12) which starts with a Conditional Start Event named cattle is slaughtered as described in Rule 13. The Surveillance is described with the WAP4 (Rule 14) indicating that a notification of the activities execution is needed. This WAP4 is refined with the Measurements of Rule 17 in the form of Business Rules. These Business Rules define how 

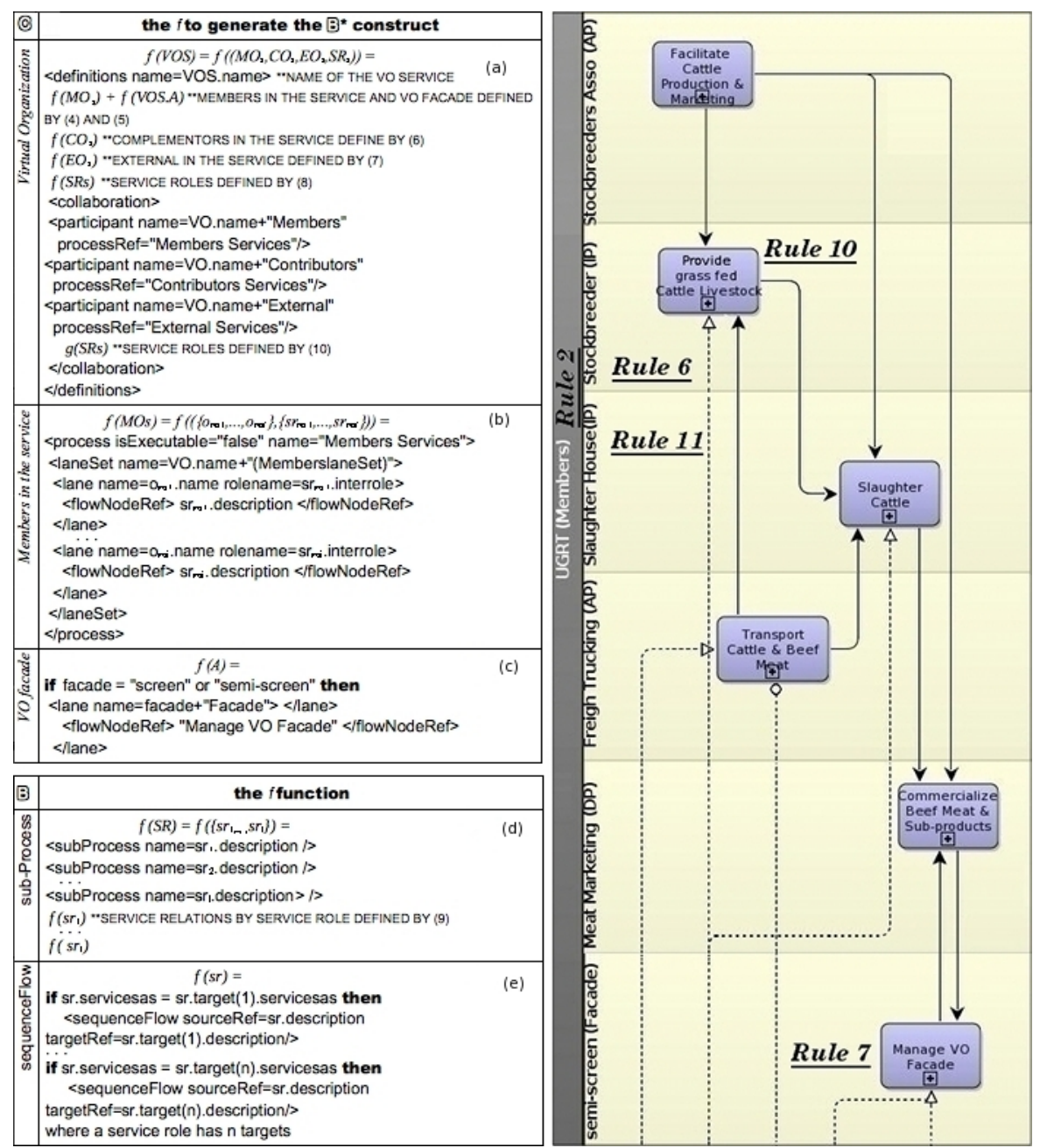

Fig. 6. Some of the Alliance Identification transformation rules

the Contribution is calculated e.g. "between $95 \%$ and $105 \%$ of the Average Cost". A second activity pattern (WAP4), is used to give the Contribution (Rule 15), respecting that Constant Surveillance is required. The rules that complement this WAP are from the Regulation Measurements of Rule 18 (e.g. "Control Figures"). An activity task is used to express the Penalization chosen in case of miss achievement, in this example is a "Warning" (Rule 20). 


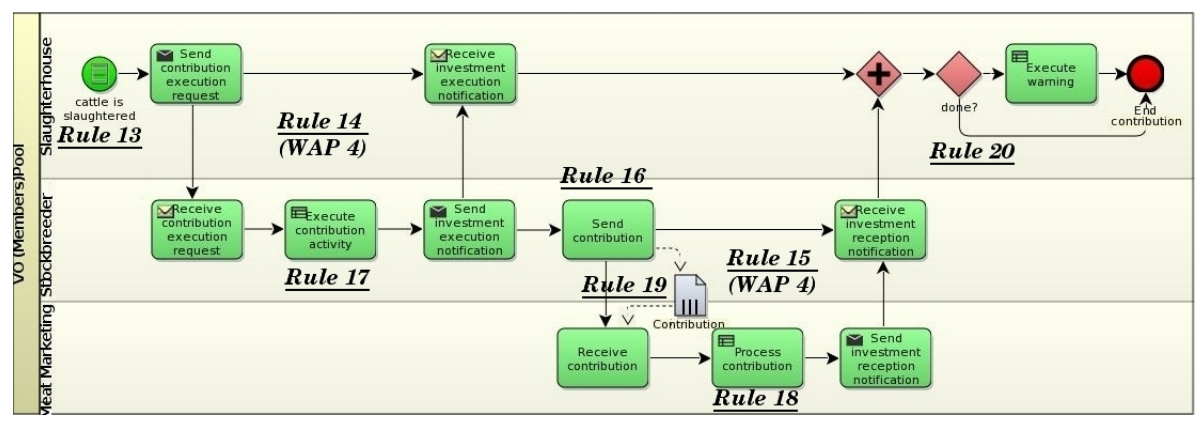

Fig. 7. The Investments of the Collaboration Willingness transformation rules

\section{Related Work}

$i^{*}$, KAOS, Map, scenarios and $\mathrm{e}^{3}$ value are well known RE modelling approaches that have evolved and matured, they are a source of many publications and offer tools as compiled in [10|2|14]. Business processes are usually developed with no reference to intentional models. Nevertheless, several works argue the need of a combination of intentional models and BPMN. [7] applies i* to express changes during the business process life cycle and [3] proposes reducing the gap between BPM and agent software paradigm. [4] determines goal satisfaction from KAOS model to business process, [16] shows how the Map formalism can capture goal achievement variability in business process models, and [21] identifies from $\mathrm{e}^{3}$ value, the value objects concerned in the business process design. Firstly, instead of adapting the existing RE modeling approaches to VOs, in this paper we relay on the $360^{\circ}$ VisiOn that as far as we know, is a novel and specifically conceived approach to guide and express requirements for VOs. Secondly, although these proposals define transformation rules, they lack of formalization. The work presented in this article offers a formalization to facilitate the automatization of the transformation process. In contrast to a manual approach and linked to the number of model elements, building an appropriate tool support saves time, avoids errors and provides standard quality BPM.

\section{Conclusions}

In this paper we reported on an approach which allows to design basic business process diagrams from information obtained at the intentional level of VOs. In particular we provided partial answers for the causals stated in the introduction:

The intentional level provides information about the problem space and the organizational level provides information about the possible solutions. We have formalized and developed a set of transformations rules principled on BPMN to assign the adequate BPMN items representations. WAPs showed to be very effective for representing single/multi participants either requesting the execution of activities or being notified about executed activities. The WAPs have also help to add more semantics and details 
for the activities description. We use the XML semantic namespace defined by [11] to generate the BPMN-XML code to increase interoperability.

At the intentional level, a prototype tool has been developed for automatically generate the graphic models from the captured instances. A new functionality will be added to automate the model transformation based on the proposed method. The intentional level includes rich information that can be used in process models (e.g. object exchange, collaboration control) that can reduce process complexity, design time at the operational level. Nevertheless, assuring model traceability remains a difficult challenge not considered in this work.

Finally, we have tested our proposal in the agroindustry and health care domains. Future work will be done to validate it in other business domains and different types of VOs with and without this transformation approach. Moreover, we are going to test how the WAPs can be adapted and/or which are the new variants to be used for representing process models executed by different types of VO and the goals defined at the intentional level. We are considering to validate the completeness and correction of the obtained BPMN diagrams by comparing process design with and without using our approach, and by verifying the obtained BPMN diagrams with companies' end users.

\section{References}

1. Basole, R.C., Rouse, W.B.: Complexity of service value networks: Conceptualisation and empirical investigation. IBM Systems Journal 47(1), 53-70 (2008)

2. Cheng, B.H.C., Atlee, J.M.: Research directions in requirements engineering. In: FOSE 2007: 2007 Future of Software Engineering, pp. 285-303. IEEE Computer Society, Washington, DC (2007)

3. Cysneiros, L.M., Yu, E.: Addressing agent autonomy in business process management-with case studies on the patient discharge process. In: Proc. 2004 IRMA Conference (2004)

4. Ghose, A.K., Koliadis, G.: Relating business process models to goal-oriented requirements models in kaos. Faculty of Informatics-Papers, p. 573 (2007)

5. Gordijn, J.: Value-based Requirements Engineering: Exploring Innovative e-Commerce Ideas. PhD thesis, Vrije Universiteit Amsterdam (2002)

6. Hickey, A.M., Davis, A.M.: Elicitation technique selection: How do experts do it. In: Proceedings of the 11th IEEE International Conference on Requirements Engineering, p. 169. IEEE Computer Society (2003)

7. Koliadis, G., Vranesevic, A., Bhuiyan, M.A., Krishna, A., Ghose, A.K.: Combining $i^{*}$ and BPMN for Business Process Model Lifecycle Management. In: Eder, J., Dustdar, S. (eds.) BPM Workshops 2006. LNCS, vol. 4103, pp. 416-427. Springer, Heidelberg (2006)

8. Li, C., Reichert, M., Wombacher, A.: On Measuring Process Model Similarity Based on High-Level Change Operations. In: Li, Q., Spaccapietra, S., Yu, E., Olivé, A. (eds.) ER 2008. LNCS, vol. 5231, pp. 248-264. Springer, Heidelberg (2008)

9. Maiden, N.A.M.: Crews-savre: Scenarios for acquiring and validating requirements. Journal for Automate Software Engineering 5(4), 419-446 (1998)

10. Nuseibeh, B., Easterbrook, S.: Requirements engineering: a roadmap. In: ICSE 2000: Proceedings of the Conference on The Future of Software Engineering, pp. 35-46. ACM, New York (2000)

11. OMG. Business process model and notation (BPMN). Technical Report 2, Object Management Group (June 2010) 
12. Parung, J., Bititci, U.S.: A conceptual metric for managing collaborative networks. Journal of Modellling in Management 1(2), 116-136 (2006)

13. Priego-Roche, L.-M., Rieu, D., Front, A.: A $360^{\circ}$ Vision for Virtual Organizations Characterization and Modelling: Two Intentional Level Aspects. In: Godart, C., Gronau, N., Sharma, S., Canals, G. (eds.) I3E 2009. IFIP AICT, vol. 305, pp. 427-442. Springer, Heidelberg (2009)

14. Priego-Roche, L.M.: Intentional and Organizational Information Systems modelling for Virtual Organizations. PhD thesis, University of Grenoble (2011)

15. Rolland, C., Prakash, N.: Bridging the gap between organisational needs and ERP functionality. Requirements Engineering Journal 5, 180-193 (2000)

16. Rolland, C., Prakash, N.: On the adequate modeling of business process families. In: 8th Workshop on Business Process Modeling, Development, and Support (BPMDS 2007), Electronic Ressource (2007)

17. Salvador, T., Scholtz, J., Larson, J.: The denver model for groupware design 5 Yeeeeee Haaaaaa? In: Conferencing Applications. CSCW 1992, CHI 1995, Denver Colorado, vol. 28(1), pp. 52-58 (1996)

18. Thom, L.H., Reichert, M., Chiao, C.M., Iochpe, C., Hess, G.N.: Inventing Less, Reusing More, and Adding Intelligence to Business Process Modeling. In: Bhowmick, S.S., Küng, J., Wagner, R. (eds.) DEXA 2008. LNCS, vol. 5181, pp. 837-850. Springer, Heidelberg (2008)

19. Thom, L.H., Reichert, M., Iochpe, C.: Activity patterns in process-aware information systems: Basic concepts and empirical evidence. International Journal of Business Process Integration and Management 4(2), 93-110 (2009)

20. van Lamsweerde, A., Darimont, R., Letier, E.: Managing conflicts in goal-driven requirements engineering. IEEE Transactions on Software Engineering 24(11), 908-926 (1998)

21. Weigand, H., Johannesson, P., Andersson, B., Bergholtz, M., Edirisuriya, A., Ilayperuma, T.: Value object analysis and the transformation from value model to process model. In: Enterprise Interoperability, pp. 55-65 (2007)

22. WFMC. Terminology \& glossary. Technical Report 3, Workflow Management Coalition, UK (February 1999)

23. Yu, E.S.K.: Towards modeling and reasoning support from early-phase requirements engineering. In: IEEE 3th International Symposium on Requirements Engineering, Annapolis MD, Janvier 5-8, pp. 226-235 (1997) 